\title{
Aldoxime Dehydratase Mutants as Improved Biocatalysts for a Sustainable Synthesis of Biorenewables-Based 2-Furonitrile
}

\author{
Ji Eun Choi ${ }^{1} \mathbb{D}$, Suguru Shinoda ${ }^{2}$, Yasuhisa Asano ${ }^{2, *}$ and Harald Gröger ${ }^{1, *(\mathbb{D}}$ \\ 1 Chair of Industrial Organic Chemistry and Biotechnology, Faculty of Chemistry, Bielefeld University, \\ Universitätsstr. 25, 33615 Bielefeld, Germany; jchoi@uni-bielefeld.de \\ 2 Biotechnology Research Center, Toyama Prefectural University, 5180 Kurokawa, Imizu, Toyama 939-0398, \\ Japan; shinoda@pu-toyama.ac.jp \\ * Correspondence: asano@pu-toyama.ac.jp (Y.A.); harald.groeger@uni-bielefeld.de (H.G.)
}

Received: 2 February 2020; Accepted: 11 March 2020; Published: 26 March 2020

check for updates

\begin{abstract}
Furonitrile is an interesting nitrile product for the chemical industry due to its use as intermediate in the field of fine chemicals and pharmaceuticals or as a potential sweetener, as well as due to its access from biorenewables. As an alternative to current processes based on, e.g., the ammoxidation of furfural with ammonia as a gas phase reaction running at $>400{ }^{\circ} \mathrm{C}$, we recently reported an enzymatic dehydration of 2-furfuryl aldoxime being obtained easily from furfural and hydroxylamine. However, improving the catalytic properties of the aldoxime dehydratase biocatalyst from Rhodococcus sp. YH3-3 (OxdYH3-3) in terms of activity and stability remained a challenge. In this contribution, the successful development of aldoxime dehydratase OxdYH3-3 mutants that were generated by directed evolution and its enhanced activity toward 2-furfuryl aldoxime is reported. The mutant OxdYH3-3 N266S showed an improved activity of up to six times higher than the wild type when utilizing a substrate concentration of 50-100 mM of 2-furfuryl aldoxime.
\end{abstract}

Keywords: Aldoxime dehydratase; directed evolution; high-throughput screening; 2-furfuryl aldoxime; 2-furonitrile

\section{Introduction}

The tasks for realizing access to industrial chemicals from biorenewables on scale include the development of approaches for aromatic compounds as well as for nitriles starting from bio-based raw materials and ideally waste-materials [1]. 2-Furonitrile, being known as intermediate in the field of fine chemicals and pharmaceuticals or as a potential sweetener (due to an about thirty-fold higher sweetening power compared to sucrose) [2], combines these two compound classes. As for aromatic compounds within a bio-based economy, the furan moiety is regarded to be of particular importance because this heteroaromatic framework can be directly constructed in a substituted form from readily available sugars such as pentoses and hexoses, thus leading to 2-furfural and 5-hydroxymethylfurfural as the most prominent representatives [3]. The availability of 2-furfural from natural waste sources makes its utilization attractive for the synthesis of derivatives with industrial potential [1,3]. For example, furfural is obtained by an acid-catalyzed dehydration of cellulose biomass, and its derivatives containing nitrogen atoms have interesting features, such as the ability of furfurylamine to be used in the pharmaceutical synthesis or resin industry [4-7].

However, the current synthetic approaches for 2-furonitrile are limited. For example, the preparation of 2-furonitrile from furfural through ammoxidation requires reaction temperatures exceeding $400{ }^{\circ} \mathrm{C}$ [2], whereas other procedures that are conducted on lab scale revealed to be tedious [8-12]. 
In continuation of our research on the biocatalytic synthesis of nitriles under cyanide-free conditions as an alternative synthetic technology for nitriles [13-25], we could also demonstrate a proof of concept for the two-step conversion of furfural into 2-furonitrile (Scheme 1) [24,25]. In detail, furfural (1) is transformed into its aldoxime (2) by spontaneous condensation with hydroxylamine, which is a bulk chemical that is utilized in $\alpha$-caprolactam manufacturing. Subsequently, the aldoxime is enzymatically dehydrated in an aqueous medium to furnish the desired 2-furonitrile (3).

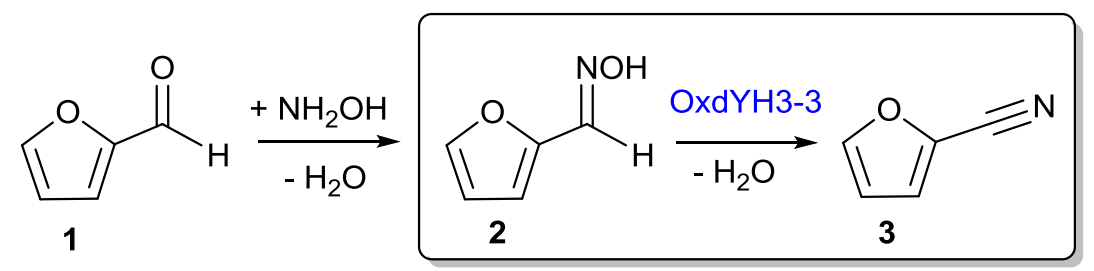

Scheme 1. Two step synthesis of 2-furonitrile from furfural based on dehydration via aldoxime dehydrate OxdYH3-3.

However, in spite of the availability of a recombinant form of an aldoxime dehydratase from Rhodococcus sp. YH3-3 (OxdYH3-3) [25], which was identified as a suitable biocatalyst for aromatic aldoximes in general and 2-furonitrile in particular, the limited enzyme activity and substrate concentration raise the demand for more active and stable biocatalysts as well as for a process running at elevated substrate loading. In the following, we report our results on the development of improved mutants of this enzyme that show an increased activity and enable an improved synthetic access to 2-furonitrile.

\section{Results and Discussion}

\subsection{High Throughput Screening of OxdYH3-3 Mutants by Using a UV Microspectrometer}

The generation of the OxdYH3-3 mutants for our study was carried out by error-prone PCR, and its potentially enhanced activity toward the target substrate 2-furfuryl aldoxime at a substrate concentration of $10 \mathrm{mM}$ was screened with microplate spectroscopy. Since under UV light, 2-furfuryl aldoxime and 2-furonitrile showed maximum absorbance at 270 and $230 \mathrm{~nm}$, respectively, the screening of the OxdYH3-3 mutants was based on measuring a decrease in absorbance at $270 \mathrm{~nm}$. The clones that showed an absorbance between 0 and 1 (lower 10\%) at $270 \mathrm{~nm}$ (Figure 1) were chosen for further studies.

$$
\text { (A) }
$$

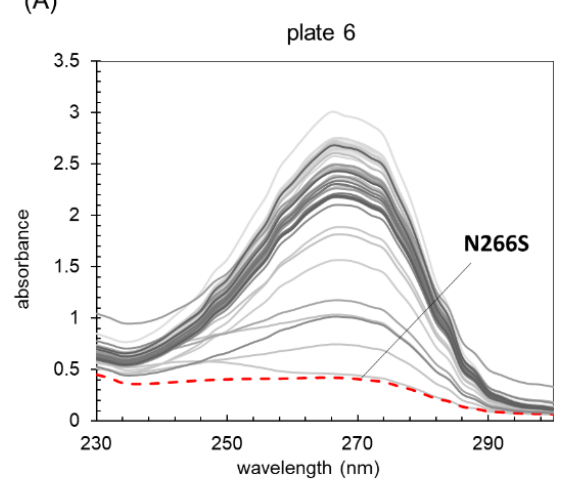

(B)

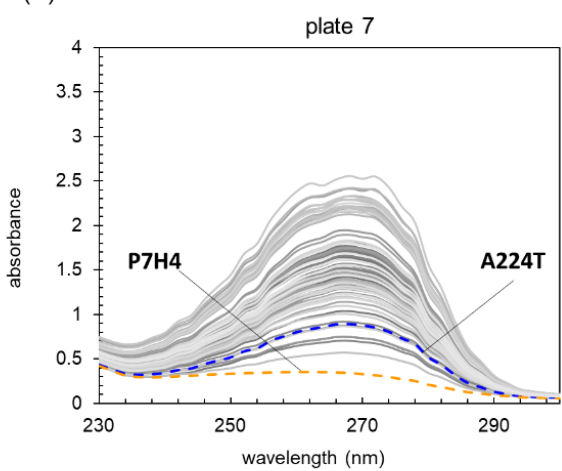

Figure 1. The UV absorbance spectrum of OxdYH3-3 mutants. OxdYH3-3 mutant clones were inoculated in 96 deep well plates, and fully-grown cells were transferred to new 96 well plates to screen OxdYH3-3 mutants that showed an improving activity to 2-furfuryl aldoxime. $10 \mathrm{mM}$ furfuryl aldoxime was added in the plate, and, after a specific time, the reaction mixture was isolated and diluted. The UV absorbance between 230 and $300 \mathrm{~nm}$ was measured. Among 10 microplates, N266S was found in plate 6 (A), P7H4 and A224T were found in plate 7 (B). 
Accordingly, the OxdYH3-3 variants N266S, A224T, and P7H4 (Val264Ala, Ile302Ser, Gln330Leu) were prioritized, followed by sequence analysis (Figure 1 ).

Subsequently, the overexpression level of OxdYH3-3 wild type (WT) and mutant proteins were analyzed through SDS-PAGE. Thick bands at $41.3 \mathrm{kDa}$ were observed, showing approximately the same high overexpression level with OxdYH3-3 WT and mutants (Figure 2).

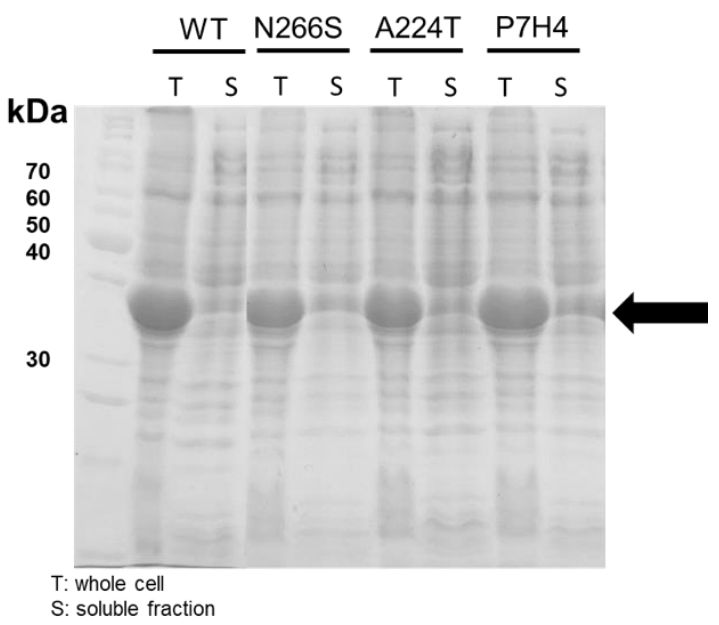

Figure 2. The expression of OxdYH3-3 wild type (WT) and its mutants. Overexpressed proteins under cultivation in an autoinduction medium was disrupted and separated into total and soluble protein following analysis by electrophoresis. The first lane was a protein size marker corresponding the number on the left side. The sizes of OxdYH3-3 WT and mutants were approximately $41.3 \mathrm{kDa}$ (arrow). $\mathrm{T}$, total protein; $\mathrm{S}$, soluble protein.

\subsection{The Improved Activity Measurement of OxdYH3-3 Mutants}

In the next steps, we gained interest in evaluating the activity of selected mutants in synthetic biotransformations. As in our previous work, the substrate concentration with the wild-type enzyme was only $10 \mathrm{mM}$ [25]. We studied these mutants in the dehydration of furfuryl aldoxime at increased 50 and $100 \mathrm{mM}$ concentrations in the presence of a biocatalyst loading of approximately $0.5 \mathrm{~g} \cdot \mathrm{L}^{-1}$ wet cells (Table 1). At first, E-3-pyridine aldoxime was used as a standard substrate since OxdYH3-3 showed the highest relative activity for E-3-pyridine aldoxime in a previous study [24,25]. At an increased substrate concentration of $100 \mathrm{mM}$ of E-3-pyridine aldoxime, OxdYH3-3 N266S showed a three times higher activity $\left(1.73 \mathrm{U} \cdot \mathrm{g}^{-1}\right.$; measured according to the conversion after $\left.1 \mathrm{~h}\right)$ compared to the one of the wild-type enzyme (0.52 U.g ${ }^{-1}$; Table 1$)$. In addition, this mutant N266S showed excellent activity for 2-furfuryl aldoxime at $100 \mathrm{mM}$ with $1.16 \mathrm{U} \cdot \mathrm{g}^{-1}$, which was four times higher than the one of the Oxd YH3-3 WT. In comparison, the mutants OxdYH3-3 A224T and P7H4 appeared to have similar or just slightly higher conversions compared to the wild-type enzyme (Table 1).

In terms of conversions, $19 \%$ and $5 \%$ were found for the wild-type of the aldoxime dehydratase, OxdYH3-3 WT, when starting from a substrate concentration of $50 \mathrm{mM}$ of E-3-pyridine aldoxime and 2-furfuryl aldoxime, respectively (Figure 3).

It is noteworthy that also in these biotransformations the mutant OxdYH3-3 N266S turned out to be superior over the wild-type enzyme and gave a three times (54\%) and eight times ( $40 \%$ ) higher conversion when comparing the data for the reactions running at $50 \mathrm{mM}$ of E-3-pyridine and 2-furfuryl aldoxime after a reaction time of $1 \mathrm{~h}$, respectively (Figure 3).

Furthermore, the conversion could be further increased by simply adding more amount of biocatalyst. For example, when the amount of catalyst was doubled, the conversion in the reaction starting from $50 \mathrm{mM}$ of 2-furfuryl aldoxime was $61 \%$ when using the whole cells containing the wild-type enzyme OxdYH3-3 WT after a reaction time of $1 \mathrm{~h}$. An even better result at such an increased 
biocatalyst loading was obtained in the presence of the mutant OxdYH3-3 N266S, which led to a full conversion under such conditions (data not shown graphically).

Table 1. The activity of OxdYH3-3 WT and mutants. The activities at $1 \mathrm{~h}$ toward 50 and $100 \mathrm{mM}$ of E-3-pyridine aldoxime and 2-furfuryl aldoxime were compared.

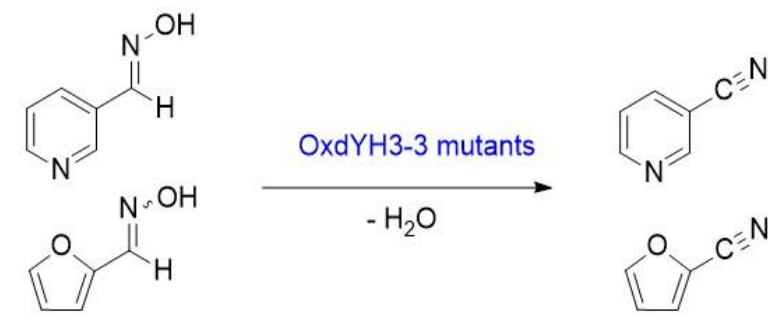

\begin{tabular}{ccccc}
\hline \multicolumn{5}{c}{$\mathbf{U}^{-\mathbf{- 1}}\left(\boldsymbol{\mu} \mathbf{m o l} \cdot \mathbf{m i n}^{-\mathbf{1}} \cdot \mathbf{g}^{-\mathbf{1}}\right)$} \\
\hline \multirow{2}{*}{ OxdYH3-3 } & \multicolumn{2}{c}{ E-3-pyridine aldoxime } & \multicolumn{2}{c}{ 2-furfuryl aldoxime } \\
\cline { 2 - 5 } & $50 \mathrm{mM}$ & $100 \mathrm{mM}$ & $50 \mathrm{mM}$ & $100 \mathrm{mM}$ \\
\hline WT & 0.62 & 0.52 & 0.15 & 0.29 \\
N266S & 1.85 & 1.73 & 1.24 & 1.16 \\
A224T & 0.68 & 0.52 & 0.37 & 0.28 \\
P7H4 & 0.81 & 0.78 & 0.53 & 0.38 \\
\hline
\end{tabular}

(A)

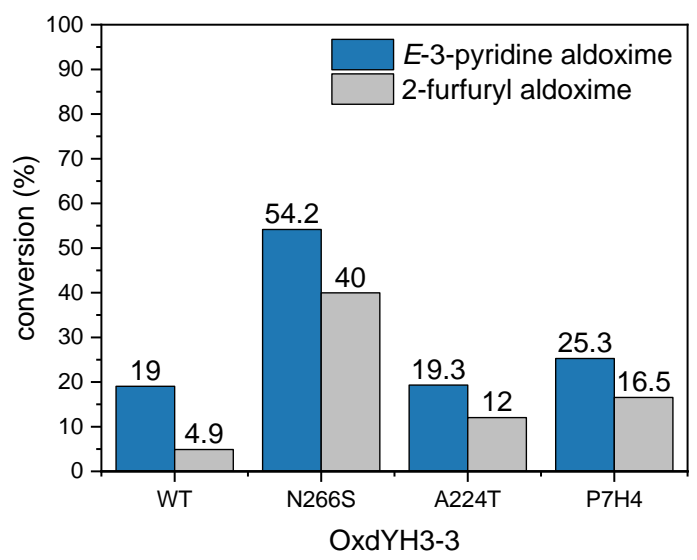

(B)

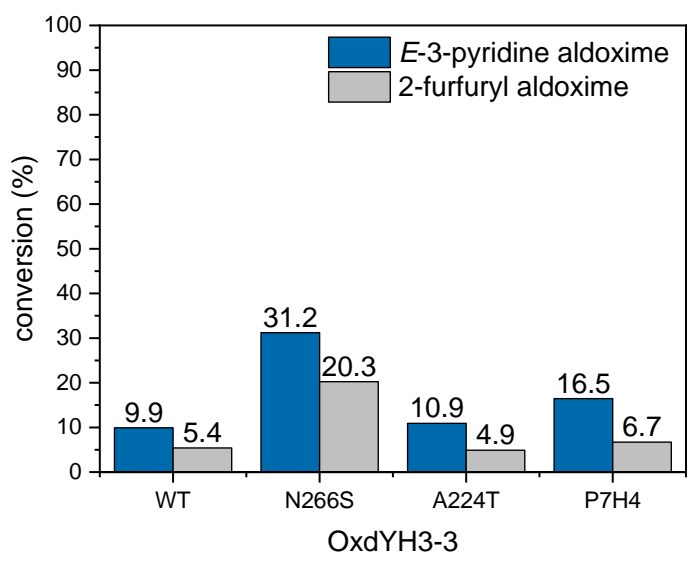

Figure 3. Biotransformations of OxdYH3-3 WT and mutants (1 h) at $50 \mathrm{mM}(\mathbf{A})$ and $100 \mathrm{mM}(\mathbf{B})$ of substrate concentration.

In addition to the improved conversions found in this biotransformation study, OxdYH3-3 N266S showed improved activity data compared to the WT enzyme. The $\mathrm{V}_{\max }$ values of OxdYH3-3 WT and $\mathrm{N} 266 \mathrm{~S}$ were 0.14 and $0.41 \mathrm{U}\left(\mu \mathrm{M} \cdot \mathrm{min}^{-1}\right)$, respectively. In addition, $\mathrm{K}_{\mathrm{m}}$ and $\mathrm{k}_{\text {cat }}$ values were $70 \mu \mathrm{M}$ and $0.37 \mathrm{~min}^{-1}$ for WT and $650 \mu \mathrm{M}$ and $2 \mathrm{~min}^{-1}$ for N266S, respectively. Even though the $\mathrm{K}_{\mathrm{m}}$ value of OxdYH3-3 N266S was much higher than the one for the WT, the catalytic efficiency of N266S was approximately five times higher than WT. In addition, kinetic data of other Oxd enzymes were determined in previous studies, and the $\mathrm{K}_{\mathrm{m}}$ values of OxdRG and OxdA towards aromatic aldoximes such as pyridine-3-aldoxime and pyridine-2-aldoxime were 20 and $3.4 \mathrm{mM}$, respectively [13-23,26,27]. Compared to these results, the $\mathrm{K}_{\mathrm{m}}$ values of OxdYH3-3 WT and N266S were significantly lower towards the aromatic aldoxime 2-furfuryl aldoxime. Furthermore, OxdRE as the enzyme with the highest homology to OxdYH3-3 WT among the Oxd enzymes, showed a higher $\mathrm{K}_{\mathrm{m}}$ value for cyclohexanecarboxaldehyde oxime (0.99 mM) than OxdYH3-3 [28]. 
It should be further added that a good correlation between the conversion determined by GC, and the change of absorbance was observed. The decrease of absorbance in the UV spectra at 245 and $270 \mathrm{~nm}$ was consistent with the decrease of E-3-pyridine and 2-furfuryl aldoxime, respectively, as measured by GC (Figure 4).

Accordingly, the mutant OxdYH3-3 N266S showed a significantly stronger decrease of the maximum absorbance of 3-pyridine aldoxime and 2-furfuryl aldoxime at 245 and $270 \mathrm{~nm}$, respectively, compared to the wild-type enzyme OxdYH3-3 WT (and other mutants), thus indicating improved catalytic abilities.

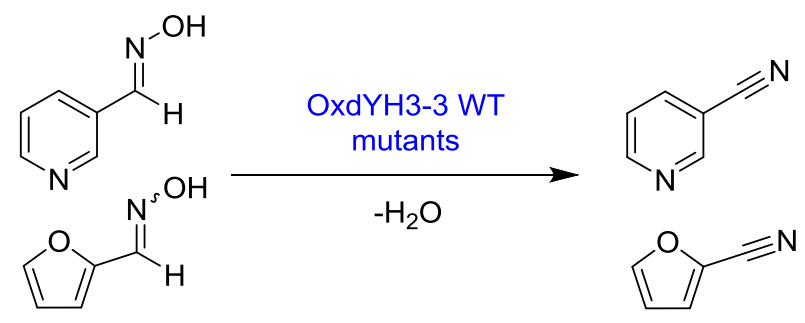

(A)

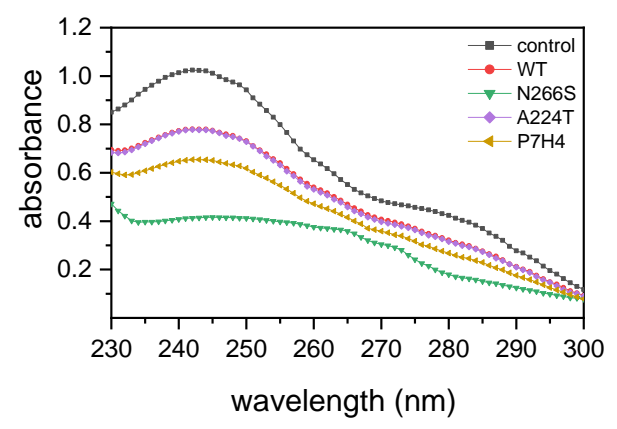

(B)

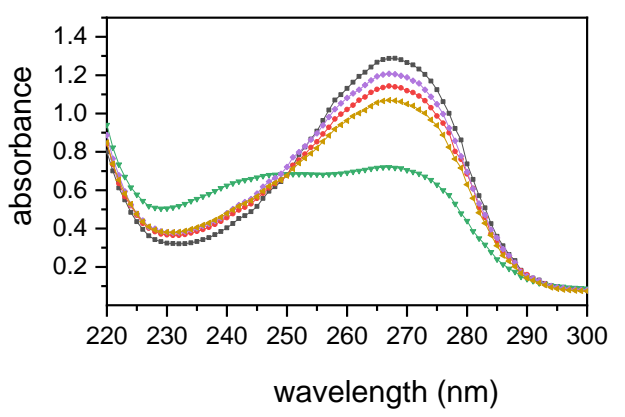

Figure 4. Absorbance scans of reaction solutions after $1 \mathrm{~h}$ of reaction time for reactions being performed at a substrate concentration of $50 \mathrm{mM}$ of E-3-pyridine aldoxime (A) and 2-furfuryl aldoxime (B). Experiments with solutions of the substrates without biocatalysts were carried out as control experiments.

Thus, this methodology for the determination of the enzymatic activities and conversion of the biotransformation through measurement of the absorbance at 245 and $270 \mathrm{~nm}$ instead HPLC, GC or thin layer chromatography represents a practical and easy-to-carry-out alternative to screen new biocatalysts and mutants towards the dehydration of various aldoximes.

\subsection{Homology Modelling of OxdYH WT and N266S}

A further goal was to rationalize the improved activity of the enzyme mutants. Toward this end, homology model structures of the wild-type enzyme OxdYH3-3 WT and the prioritized mutant N266S were constructed based on the literature-known OxdRE crystal structure [16] (PDB No. 3A17_A; co-crystalized with heme and butyl aldoxime) and a subsequent docking with $E$-2-furfuryl aldoxime by using the Autodock4 software (Figure 5).

The docking results showed that the nitrogen of $\mathrm{N}-\mathrm{OH}$ in 2-E-furfuryl aldoxime was facing Fe in the heme group (2.7 ̊̊). In addition, His320 and Ser219 in OxdYH3-3 WT and N266S formed hydrogen bonds with the oxygen atom of the $\mathrm{N}-\mathrm{OH}$ group of the 2-furfuryl aldoxime ligand. It is noteworthy that the distances of the hydrogen bonding of wild type and N266S as well as the free binding energies did not show a significant difference, which indicates that the mutation did not directly influence the interaction between the substrate and its adjacent residues (see also Supplementary Materials, Figure S3). 
(A)

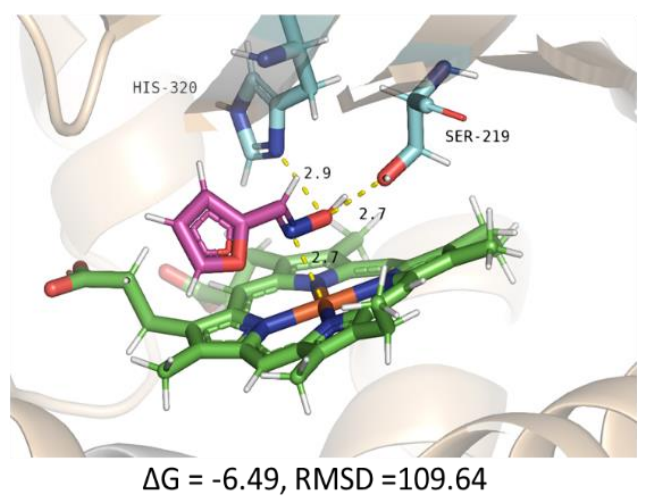

(B)

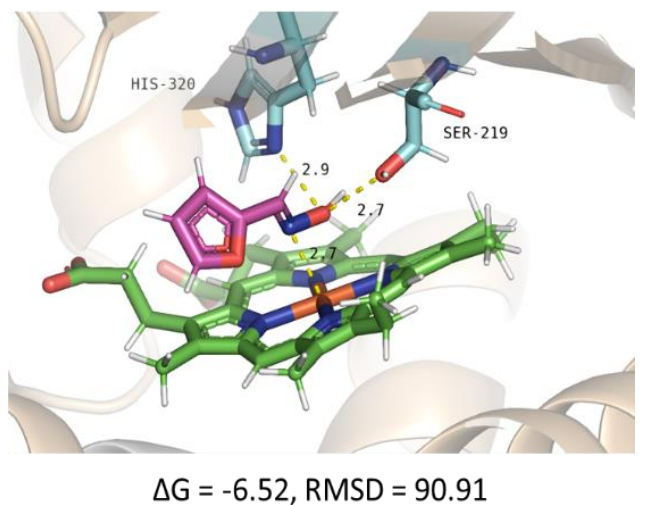

Figure 5. Binding pocket on OxdYH3-3 WT (A) and N266S (B) docking with E-2-furfuryl aldoxime. Only heme and residues His320 and Ser219, forming hydrogen bonds, are shown. Heme, E-2-furfuryl aldoxime, and the residues are shown as sticks colored in green, purple, and sky blue, respectively. The dashed lines showed distances between the atoms in a range of 2-3 $\AA$.

Nonetheless, a residues network analysis between the wild type enzyme and the mutant that used Ring2 and Cytoscape [29,30] could suggest that the long-range interaction at the remote site could have changed structure flexibility, thus leading to the observed beneficial effects (see Supplementary Materials, Figure S4).

\section{Materials and Methods}

\subsection{Materials and Preparation of OxdYH3-3 Transformants}

Chemicals including E-3-pyridine aldoxime, 3-cyanopyridine, 2-furaldehyde, and 2-furonitrile were purchased from Sigma Aldrich (Germany). OxdYH3-3 WT in a pET28b vector was constructed and transformed into BL21(DE3) Escherichia coli cell followed by methods in the previous study [25].

\subsection{Synthesis of 2-Furfuryl Aldoxime}

2-Furfuryl aldoxime was synthesized from 2-furaldehyde. Toward this end, $20 \mathrm{~mL}$ of water containing 2-furaldehyde ( $120.72 \mathrm{mmol})$ was mixed with $144.86 \mathrm{mmol} \mathrm{NH} \mathrm{H}_{2} \mathrm{OH} \cdot \mathrm{HCl}$. Sodium carbonate (72.43 mmol) dissolved in $30 \mathrm{~mL}$ of water was added dropwise. After overnight reaction, it was extracted by adding the same volume of ethyl acetate three times. The organic solution was washed with water and dried by magnesium sulphate. Yellow powder was obtained after evaporating the solvent with a rotary evaporator. The ${ }^{1} \mathrm{H}-\mathrm{NMR}$ spectra and ESI mass spectra were measured (see Supplementary Materials).

\subsection{Construction of Mutant Library by Error-Prone PCR}

The mutant library was constructed by using a PCR random mutagenesis kit (GeneMorph II Random Mutagenesis Kit, Agilent) followed the manual provided by a manufacturer with modification. The forward primer was a T7 promoter primer (5'-TAATACGACTCACTATAGGG-3'), and the reverse primer was a $\mathrm{T} 7$ terminator primer ( $5^{\prime}$-GCTAGTTATTGCTCAGCGG-3'). To be specific, error-prone PCR was performed in $50 \mu \mathrm{L}$ of a reaction solution containing $100 \mathrm{ng}$ of pET28b-OxdYH3-3 as a template, $1 \mu \mathrm{L}$ of titanium Taq polymerase, $640 \mu \mathrm{M}$ of $\mathrm{MgSO}_{4}, 40 \mu \mathrm{M}$ of dGTP, $0.1 \mu \mathrm{M}$ of of each primer, $1 \mu \mathrm{L}$ dNTP mix, and $5 \mu \mathrm{L}$ of a titanium PCR buffer. The PCR program consisted of 25 cycles of $94^{\circ} \mathrm{C}$ for $30 \mathrm{~s}, 45^{\circ} \mathrm{C}$ for $1.5 \mathrm{~min}$, and $68^{\circ} \mathrm{C}$ for $1 \mathrm{~min}$. The amplified DNA fragment was purified and cloned into the $\mathrm{NcoI}$ and HindIII sites of the pET28b vector (Novagen) by using an in-fusion HD Cloning Kit (Takara) to obtain pET28b-OxdYH3-3 for expression as a C-terminal His-tagged protein. The plasmid pET28b-OxdYH3-3 was transformed into BL21(DE3) and spread on the LB plate containing antibiotics. For mutant screening, colonies were inoculated to a $1 \mathrm{~mL}$ autoinduction medium in a 96 deep well 
plate and cultured for $18 \mathrm{~h}$ at $30^{\circ} \mathrm{C}$. Twenty microliters of cell suspension were transferred to a 96 deep well plate containing fresh $980 \mu \mathrm{L}$ of an autoinduction medium and cultivated for $20 \mathrm{~h}$ at $30^{\circ} \mathrm{C}$.

\subsection{Screening of OxdYH3-3 WT and Mutants by of UV Spectrum Scan}

OxdYH3-3 was overexpressed in an autoinduction medium containing $0.2 \%$ glucose, $0.5 \%$ lactose, $1.2 \%$ tryptone, $2.4 \%$ yeast extract, $0.5 \%$ glycerol, and phosphate $\left(0.17 \mathrm{M} \mathrm{KH}_{2} \mathrm{PO}_{4}\right.$ and $\left.0.72 \mathrm{M} \mathrm{K}_{2} \mathrm{HPO}_{4}\right)$, and the cell was harvested by centrifugation at $4{ }^{\circ} \mathrm{C}, 4,000 \mathrm{rpm}$ for $30 \mathrm{~min}$ after $20 \mathrm{~h}$ of shaking incubation at $30^{\circ} \mathrm{C}$ for $20 \mathrm{~h}$. The cell pellet was washed by a $50 \mathrm{mM}$ phosphate buffer ( $\mathrm{pH} \mathrm{7.0)}$ and resuspended in a $1 \mathrm{~mL}$ phosphate buffer. A $190 \mu \mathrm{L}$ cell suspension was transferred to 96 well plates, and $10 \mu \mathrm{L}$ of substrates ( $200 \mathrm{mM}$ dissolved in DMSO) were added. After 3 and $20 \mathrm{~h}$ incubation at $30^{\circ} \mathrm{C}$, the reaction mixture was centrifuged at 10,000 rpm for $10 \mathrm{~min}$ to remove the cell pellet, and 5-10 $\mu \mathrm{L}$ solutions were transferred to clean 96 well following a 10-20 fold-dilution with a phosphate buffer to measure UV absorbance. The enhanced activity toward 2-furfuryl aldoxime of mutated OxdYH3-3 was screened by measuring UV absorbance (Biorad). The absorbance of 2-furfuryl aldoxime and 2-furonitrile were scanned from 230 to $350 \mathrm{~nm}$ to determine the specific wavelength that showed the biggest difference between aldoxime and nitrile.

\subsection{Analysis of the DNA Sequence of OxdYH3-3 Mutants}

Selected mutants were subjected to DNA sequence analysis. The mutant clones transformed into BL21(DE3) were spread on an LB-agar plate containing $50 \mu \mathrm{g} \cdot \mathrm{mL}^{-1}$ kanamycin and incubated at $37^{\circ} \mathrm{C}$ for $20 \mathrm{~h}$. The single colony was inoculated in $5 \mathrm{~mL}$ of an LB broth and cultured for overnight at $37^{\circ} \mathrm{C}$ in the shaking incubator at $150 \mathrm{rpm}$. The plasmid was isolated by using the plasmid isolated kit (Analytik Jena), and nucleotide sequences were analyzed by ABI 3730XL sequencing machines with the modified Sanger method (Eurofins Genomics, Germany).

\subsection{Protein Purification}

Histidine-tagged OxdYH3-3 WT and N266S were purified by using nickel resin affinity chromatography. Ni-NTA agarose resin was purchased from Qiagen. The soluble fraction from lysis was loaded on 1-3 mL of Ni-NTA resin and incubated for $15 \mathrm{~min}$ following washing with $100 \mathrm{~mL}$ of a washing buffer ( $50 \mathrm{mM} \mathrm{NaH}_{2} \mathrm{PO}_{4}, 300 \mathrm{mM} \mathrm{NaCl}, 20 \mathrm{mM}$ imidazole, $\mathrm{pH}$ 8.0) and elution with $10 \mathrm{~mL}$ of an elution buffer ( $50 \mathrm{mM} \mathrm{NaH} \mathrm{PO}_{4}, 300 \mathrm{mM} \mathrm{NaCl}, 500 \mathrm{mM}$ imidazole, $\mathrm{pH}$ 8.0). The buffer was changed to a sodium phosphate buffer ( $\mathrm{pH} 7.0,50 \mathrm{mM})$ after purification by using a PD-10 desalting column (GE Healthcare), and it was kept in a $4{ }^{\circ} \mathrm{C}$ freezer.

\subsection{Characterization of Purified OxdYH3-3 Catalyst}

A reaction solution containing 2-furfuryl aldoxime was prepared through the dilution of a DMSO stock solution $(1 \mathrm{M})$ with a phosphate buffer $(\mathrm{pH} 7.0,50 \mathrm{mM})$. Twenty microliters of purified enzyme was added to $80 \mu \mathrm{L}$ of a substrate solution in 96 well microplates. The decreases of absorbance at $270 \mathrm{~nm}$ were measured over time at $30^{\circ} \mathrm{C}$. The extinction coefficient was determined by mixing the $10 \mu \mathrm{L}$ of a $0.01-100 \mathrm{mM}$ stock solution with $990 \mu \mathrm{L}$ of a phosphate buffer, and $20 \mu \mathrm{L}$ of the mixture was transferred again to measure UV absorbance. The regression curve was drawn with Excel, and the extinction coefficient was calculated based on the Beer-Lambert equation. The calculated extinct coefficient of 2-furfuryl aldoxime was $17.4 \mathrm{mM}^{-1} \cdot \mathrm{cm}^{-1}$. The $K_{m}$ was determined by Origin program by using Michaelis-Menten equation. One unit is defined as $\mu \mathrm{M} \cdot \mathrm{min}^{-1}$ (see Supplementary Materials, Figure S2).

\subsection{Conversion of Aldoximes to Corresponding Nitriles by Using OxdYH3-3 WT and Mutants}

The transformants were inoculated to $5 \mathrm{~mL}$ of a TB (terrific broth) medium containing $50 \mu \mathrm{g} \cdot \mathrm{mL}^{-1}$ kanamycin. After overnight cultivation at $37^{\circ} \mathrm{C}$, the culture was re-inoculated to $50 \mathrm{~mL}$ of an 
autoinduction medium containing $0.2 \%$ glucose, $0.5 \%$ lactose, $1.2 \%$ tryptone, $2.4 \%$ yeast extract, $0.5 \%$ glycerol, and phosphate $\left(0.17 \mathrm{M} \mathrm{KH}_{2} \mathrm{PO}_{4}\right.$ and $\left.0.72 \mathrm{M} \mathrm{K}_{2} \mathrm{HPO}_{4}\right)$ and kept at $30{ }^{\circ} \mathrm{C}$ up to $18 \mathrm{~h}$ with shaking. The overexpressed cell was collected and washed with distilled water once. The pellet was resuspended to $5 \mathrm{~mL}$ of a phosphate buffer $(\mathrm{pH} 7.0,50 \mathrm{mM})$ and stored at $4{ }^{\circ} \mathrm{C}$ in a freezer until it was used in the next experiment. The overexpression level was analyzed by the SDS-PAGE of the whole cell.

A volume of $100 \mu \mathrm{L}$ of 3-pyridine aldoxime and 2-furfuryl aldoxime (0.5 and $1 \mathrm{M}$ in DMSO, respectively) were added to a $900 \mu \mathrm{L}$ cell suspension. The mixtures were incubated in a shaker at $30^{\circ} \mathrm{C}$ with $1000 \mathrm{rpm}$ for $1 \mathrm{~h}$, and a sample of $100 \mu \mathrm{L}$ reaction mixture was taken to measure GC. A volume of $20 \mu \mathrm{L}$ of $1 \mathrm{M} \mathrm{NaOH}$ and $180 \mu \mathrm{L}$ of ethyl acetate was added to the reaction solution to inactivate the enzyme. The reaction mixture was centrifuged to isolate the organic phase, which was subjected to GC measurement. At the same time, $100 \mu \mathrm{L}$ of the reaction mixture was isolated and centrifuged. The clear supernatant was diluted 20 times with a phosphate buffer $(\mathrm{pH} 7.0,50 \mathrm{mM})$ and transferred to a 96 microwell plate to measure spectrophotometry.

On the other hand, $9.15 \mathrm{~g}$ of OxdYH3-3 WT and $8.24 \mathrm{~g}$ of N266S of wet cells were resuspended in a phosphate buffer ( $\mathrm{pH} 7.0,50 \mathrm{mM}$ ) to make $15 \mathrm{~mL}$. A two-milliliter solution was taken to measure activity by using the whole cell. An activity-using aldoxime was measured with the same procesure as above.

\subsection{Determination of Conversion from Aldoximes to Nitrile by GC}

The conversion was determined by GC measurement (Shimadzu GC-2010 plus). GC conditions were optimized to efficiently separate the products from the reactants in a Phenomenex ZB-5MSi capillary column $(30 \mathrm{~m} \times 0.25 \mathrm{~mm} \times 0.25 \mu \mathrm{m})$. Nitrogen was used as carrier gas at a flow rate of $2.25 \mathrm{~mL} \mathrm{~min}^{-1}$ with an average velocity of $46.9 \mathrm{~cm} \mathrm{~s}^{-1}$ and a pressure of $147.6 \mathrm{kPa}$. The analysis run was started with an oven temperature of $60{ }^{\circ} \mathrm{C}$ that ramped to $250{ }^{\circ} \mathrm{C}$ at $15^{\circ} \mathrm{C} \mathrm{min}{ }^{-1}$.

\subsection{Measurement of the UV Spectrum of Aldoximes and Nitriles After the Reaction}

The enhanced activity toward 3-E-pyridine aldoxime and 2-furfuryl aldoxime of OxdYH3-3 was screened by measuring UV absorbance. The absorbance of 3-E-pyridine aldoxime, 3-cyanopyridine, 2-furfuryl aldoxime, and 2-furonitrile were scanned from 200 to $300 \mathrm{~nm}$ to observe the difference between aldoxime and nitrile as a time course. After the reaction supernatant was diluted with a phosphate buffer ( $\mathrm{pH} 7.0,50 \mathrm{mM}$ ) from 10 to 100 times, the absorbance was determined with a microplate spectrophotometer (Tecan).

\subsection{Homology Modelling}

The homology models of OxdYH3-3 WT and N266S were created by SWISS-MODEL and Modeller using OxdRE which contains heme and 1-Z-butanal oxime as a template and following verification by QMEAN server and ERRAT in ERRAT server [16,31-33]. The modelling that was created by SWISS-MODEL showed high scores within verification; therefore, this model was used for further study. The structure optimization of 2-E-furfuryl aldoxime was done by Avogadro [34]. Molecular docking studies with OxdYH3-3 WT and N266S were performed by Autodock4 and visualized by autodock tools and PyMOL [35]. The hydrogen bonds between ligands adjacent residues were analyzed with LIGPLOT program [36]. Models that were chosen for the next step was subjected to an analysis of residues network that used Ring 2.0 and Cytoscape [29,30] and a further analysis that used the UCSF Chimera program [37].

\section{Conclusions}

In conclusion, the successful development of mutants of the recombinant aldoxime dehydratase OxdYH3-3 in E.coli BL21(DE3) that were generated by directed evolution and its enhanced activity toward 2-furfuryl aldoxime has been reported. Approximately 1000 mutant clones were constructed 
by random mutagenesis, and the primary screening was done by measuring absorbance at $270 \mathrm{~nm}$, which corresponded to E-2-furfuryl aldoxime. The prioritized mutant OxdYH3-3 N266S showed an improved activity of up to six times higher than the wild type when utilizing a substrate concentration of 50-100 mM of 2-furfuryl aldoxime, thus furnishing 2-furonitrile being a valuable nitrile product for the chemical industry due to its use as intermediate in the field of fine chemicals and pharmaceuticals or as potential sweetener, as well as due to its access from biorenewables. Interestingly, the mutation of this improved biocatalyst OxdYH3-3 N266S is located at a remote site that is far away from the active pocket. Among the next steps for future research work is the optimization of this biotransformation towards a process running at high substrate loading, thus fulfilling requirements of an industrially attractive application.

Supplementary Materials: The following are available online at http://www.mdpi.com/2073-4344/10/4/362/s1, Additional result of NMR and ESI-MS spectra, Figure S1: Alignment of translated polypeptide sequences of OxdYH3-3 WT and mutants, Figure S2: Michaelis-Menten plot of OxdYH3-3 WT (A) and N266S (B) towards 2-furfuryl aldoxime. Figure S3: Diagram of interactions between OxdYH3-3 WT (A) and N266S (B) to E-2-furfuryl aldoxime. The purple and yellow line showed ligand and non-ligand bond, respectively. The green dashed line indicates a hydrogen bond and its length. Protein residues involving hydrophobic contacts were shown as a red half circle with spikes, Figure S4: Residue interaction networks (RINs) of nonmutated residue Asn266 from OxdYH3-3 WT ((A) and (B)) and mutated residue Asn266Ser from N266S ((C) and (D)). Pink nobs interact residues with Asn266 and Asn266Ser, and yellow nobs are the residues directed connected to them. Asn266 in OxdYH3-3 WT interacts with Asp60, Thr82, Leu276, and Leu81 through a hydrogen bond, and Tyr278 through Van der Waals interaction which forming interaction with Ser219 (A), and it was visualized as 3D structure (B). Ball structure shows Ans266, and sticks are networking residues with Asn266 within $5 \AA$ of ligand. The blue stick is E-2-furfuryl aldoxime. On the other hand, Ans266Ser in OxdYH3-3 N266S shows only two hydrogen bond interactions with Leu276 and Leu 274 and no other types of interactions (C), suggesting no interaction with residues in the binding pocket (D).

Author Contributions: Conceptualization, supervision, funding acquisition, and project administration by H.G. and Y.A.; methodology, investigation, and writing-original draft preparation, J.E.C. and H.G.; formal analysis and visualization, J.E.C. and S.S.; writing-review and editing, H.G. and Y.A. All authors have read and agreed to the published version of the manuscript.

Funding: This research was funded by a grant-in-aid for Scientific Research (S) from the Japan Society for Promotion of Sciences (Grant No. 17H06169) and the joint DAAD-JSPS funding program "PPP Japan 2017" (DAAD grant number: 57345566).

Acknowledgments: We gratefully acknowledge generous support from the German Academic Exchange Service (DAAD) and Japan Society for the Promotion of Science (JSPS).

Conflicts of Interest: The authors declare no conflict of interest.

\section{References}

1. Dominguez de Maria, P. Industrial Biorenewables: A Practical Viewpoint; Wiley: Hoboken, NJ, USA, 2016.

2. Jennings, T.J. Process for Preparing Furonitrile. U.S. Patent 3,260,731, 1964.

3. Bohre, A.; Dutta, S.; Saha, B.; Abu-Omar, M.M. Upgrading furfurals to drop-in biofuels: An overview. ACS Sustain. Chem. Eng. 2015, 3, 1263-1277. [CrossRef]

4. Gandini, A.; Belgacem, M.N. Furans in polymer chemistry. Prog. Polym. Sci. 1997, 22, 1203-1379. [CrossRef]

5. Chatterjee, M.; Ishizaka, T.; Kawanami, H. Reductive amination of furfural to furfurylamine using aqueous ammonia solution and molecular hydrogen: An environmentally friendly approach. Green Chem. 2016, 18, 487-496. [CrossRef]

6. Nishimura, S.; Mizuhori, K.; Ebitani, K. Reductive amination of furfural toward furfurylamine with aqueous ammonia under hydrogen over Ru-supported catalyst. Res. Chem. Intermed. 2016, 42, 19-30. [CrossRef]

7. Dunbabin, A.; Subrizi, F.; Ward, J.M.; Sheppard, T.D.; Hailes, H.C. Furfurylamines from biomass: Transaminase catalysed upgrading of furfurals. Green Chem. 2017, 19, 397-404. [CrossRef]

8. Yang, S.H.; Chang, S. Highly efficient and catalytic conversion of aldoximes to nitriles. Org. Lett. 2001, 3, 4209-4211. [CrossRef]

9. Bandgar, B.P.; Makone, S.S. Organic reactions in water: Transformation of aldehydes to nitriles using NBS under mild conditions. Synth. Commun. 2006, 36, 1347-1352. [CrossRef] 
10. Narsaiah, A.; Sreenu, D.; Nagaiah, K. Direct oxidative conversion of alcohols, aldehydes and amines into nitriles using hypervalent iodine(III) reagent. Synth. Commun. 2006, 36, 137-140. [CrossRef]

11. Yadav, L.D.S.; Srivastava, V.P.; Patel, R. Bromodimethylsulfonium bromide (BDMS): A useful reagent for conversion of aldoximes and primary amides to nitriles. Tetrahedron Lett. 2009, 50, 5532-5535. [CrossRef]

12. Sridhar, M.; Reddy, M.K.K.; Sairam, V.V.; Raveendra, J.; Godala, K.R.; Narsaiah, C.; Ramanaiah, B.C.; Reddy, C.S. Acetohydroxamic acid: A new reagent for efficient synthesis of nitriles directly from aldehydes using $\mathrm{Bi}(\mathrm{OTf})_{3}$ as the catalyst. Tetrahedron Lett. 2012, 53, 3421-3424. [CrossRef]

13. Asano, Y.; Kato, Y. Z-Phenylacetaldoxime degradation by a novel aldoxime dehydratase from Bacillus sp. strain OxB-1. FEMS Microbiol. Lett. 1998, 158, 185-190. [CrossRef]

14. Kato, Y.; Nakamura, K.; Sakiyama, H.; Mayhew, S.G.; Asano, Y. Novel heme-containing lyase, phenylacetaldoxime dehydratase from Bacillus sp. strain OxB-1: Purification, characterization, and molecular cloning of the gene. Biochemistry 2000, 39, 800-809. [CrossRef] [PubMed]

15. Kato, Y.; Ooi, R.; Asano, Y. Distribution of aldoxime dehydratase in microorganisms. Appl. Environ. Microbiol. 2000, 66, 2290-2296. [CrossRef] [PubMed]

16. Sawai, H.; Sugimoto, H.; Kato, Y.; Asano, Y.; Shiro, Y.; Aono, S. X-ray crystal structure of michaelis complex of aldoxime dehydratase. J. Biol. Chem. 2009, 284, 32089-32096. [CrossRef] [PubMed]

17. Yamaguchi, T.; Asano, Y. Draft genome sequence of an aldoxime degrader, Rhodococcus sp. strain YH3-3. Genome Announc. 2016, 4, 406-416. [CrossRef] [PubMed]

18. Metzner, R.; Okazaki, S.; Asano, Y.; Gröger, H. Cyanide-free enantioselective synthesis of nitriles: Synthetic proof of a biocatalytic concept and mechanistic insights. ChemCatChem 2014, 6, 3105-3109. [CrossRef]

19. Betke, T.; Rommelmann, P.; Oike, K.; Asano, Y.; Gröger, H. Cyanide-free and broadly applicable enantioselective synthetic platform for chiral nitriles through a biocatalytic approach. Angew. Chem. Int. Ed. 2017, 56, 12361-12366. [CrossRef]

20. Betke, T.; Maier, M.; Gruber-Wölfler, H.; Gröger, H. Biocatalytic production of adiponitrile and related aliphatic linear $\alpha, \omega$-dinitriles. Nat. Commun. 2018, 9, 5112. [CrossRef]

21. Hinzmann, A.; Glinski, S.; Worm, M.; Gröger, H. Enzymatic synthesis of aliphatic nitriles at a substrate loading of up to $1.4 \mathrm{~kg} / \mathrm{L}$ : A biocatalytic record achieved with a heme protein. J. Org. Chem. 2019, 84, 4867-4872. [CrossRef]

22. Plass, C.; Hinzmann, A.; Terhorst, M.; Brauer, W.; Oike, K.; Yavuzer, H.; Asano, Y.; Vorholt, A.J.; Betke, T.; Gröger, H. Approaching bulk chemical nitriles from alkenes: A hydrogen cyanide-free approach through a combination of hydroformylation and biocatalysis. ACS Catal. 2019, 9, 5198-5203. [CrossRef]

23. Betke, T.; Higuchi, J.; Rommelmann, P.; Oike, K.; Nomura, T.; Kato, Y.; Asano, Y.; Gröger, H. Biocatalytic synthesis of nitriles through dehydration of aldoximes: The substrate scope of aldoxime dehydratases. ChemBioChem 2018, 19, 768-779. [CrossRef] [PubMed]

24. Kato, Y.; Ooi, R.; Asano, Y. A new enzymatic method of nitrile synthesis by Rhodococcus sp. strain YH3-3. J. Mol. Catal. B Enzym. 1999, 6, 249-256. [CrossRef]

25. Choi, J.E.; Shinoda, S.; Inoue, R.; Zheng, D.; Gröger, H.; Asano, Y. Cyanide-free synthesis of an aromatic nitrile from a biorenewable-based aldoxime: Development and application of a recombinant aldoxime dehydratase as a biocatalyst. Biocat. Biotransf. 2019, 37, 414-420. [CrossRef]

26. Oinuma, K.I.; Hashimoto, Y.; Konishi, K.; Goda, M.; Noguchi, T.; Higashibata, H.; Kobayashi, M. Novel aldoxime dehydratase involved in carbon-nitrogen triple bond synthesis of Pseudomonas chlororaphis B23 sequencing, gene expression, purification, and characterization. J. Bio. Chem. 2003, 278, 29600-29608. [CrossRef] [PubMed]

27. Xie, S.X.; Kato, Y.; Komeda, H.; Yoshida, S.; Asano, Y. A gene cluster responsible for alkylaldoxime metabolism coexisting with nitrile hydratase and amidase in Rhodococcus globerulus A-4. Biochemistry 2003, 42, 12056-12066. [CrossRef] [PubMed]

28. Kato, Y.; Yoshida, S.; Xie, S.X.; Asano, Y. Aldoxime dehydratase co-existing with nitrile hydratase and amidase in the iron-type nitrile hydratase-producer Rhodococcus sp. N-771. J. Biosci. Bioeng. 2004, 97, 250-259. [CrossRef]

29. Piovesan, D.; Minervini, G.; Tosatto, S.C.E. The RING 2.0 web server for high quality residue interaction networks. Nucleic Acids Res. 2016, 44, 367-374. [CrossRef] 
30. Shannon, P.; Markiel, A.; Ozier, O.; Baliga, N.S.; Wang, J.T.; Ramage, D.; Amin, N.; Schwikowski, B.; Ideker, T. Cytoscape: A software environment for integrated models of biomolecular interaction networks. Genome Res. 2003, 13, 2498-2504. [CrossRef]

31. SWISS-MODEL. Available online: http://swissmodel.expasy.org (accessed on 13 February 2019).

32. Eswar, N.; Eramian, D.; Webb, B.; Shen, M.Y.; Sali, A. Protein structure modeling with MODELLER. In Structural Proteomics; Kobe, B., Guss, M., Huber, T., Eds.; Methods in Molecular Biology ${ }^{\mathrm{TM}}$; Humana Press: Totowa, NJ, USA, 2008; Volume 426, pp. 145-149.

33. Colovos, C.; Yeates, T.O. Verification of protein structures: Patterns of nonbonded atomic interactions. Protein Sci. 1993, 2, 1511-1519. [CrossRef]

34. Hanwell, M.D.; Curtis, D.E.; Lonie, D.C.; Vandermeersch, T.; Zurek, E.; Hutchison, G.R. Avogadro: An advanced semantic chemical editor, visualization, and analysis platform. J. Cheminform. 2012, 4, 17. [CrossRef]

35. Morris, G.M.; Huey, R.; Lindstrom, W.; Sanner, M.F.; Belew, R.K.; Goodsell, D.S.; Olson, A.J. AutoDock4 and AutoDockTools4: Automated docking with selective receptor flexibility. J. Comput. Chem. 2009, 30, 2785-2791. [CrossRef] [PubMed]

36. Wallace, A.C.; Laskowski, R.A.; Thornton, J.M. Ligplot: A program to generate schematic diagrams of protein-ligand interactions. Protein Eng. Des. Sel. 1995, 8, 127-134. [CrossRef] [PubMed]

37. Pettersen, E.F.; Goddard, T.D.; Huang, C.C.; Couch, G.S.; Greenblatt, D.M.; Meng, E.C.; Ferrin, T.E. UCSF Chimera-A visualization system for exploratory research and analysis. J. Comput. Chem. 2004, 25, 1605-1612. [PubMed]

(C) 2020 by the authors. Licensee MDPI, Basel, Switzerland. This article is an open access article distributed under the terms and conditions of the Creative Commons Attribution (CC BY) license (http://creativecommons.org/licenses/by/4.0/). 